\title{
Development and Implementation of a Biometrics Device Design Project in an Introductory BME Course to Support Student Wellness
}

\author{
Isabel Miller, Sara Lamer, Aidan Brougham-Cook, \\ Karin J. Jensen, and Holly M. Golecki 10 \\ Department of Bioengineering, University of Illinois Urbana-Champaign, Urbana, IL, USA \\ (Received 14 August 2021; accepted 4 December 2021; published online 3 January 2022)
}

\begin{abstract}
Mental health challenges have been rising across college campuses. To destigmatize wellness practices and promote student mental health, we present a novel technical project in an introductory bioengineering course that explores stress management techniques through physiology, biosensors, and design. We hypothesize that if students measure objective, physiologic impacts of stress management techniques on themselves, they may be more likely to realize the benefits and use those techniques when needed. Additionally, through this data-driven project, we aim to appeal to engineers' critical thinking nature. To support students in selecting stress management techniques for themselves, mindfulness is introduced and practiced in the course. Initial student feedback on the introduction of mindfulness into the classroom is positive. The COVID-19 pandemic has emphasized the need to focus on student wellbeing in addition to physical health. Integration of wellness into the core curriculum can normalize the use of these resources within engineering departments and colleges and equip students with stress management tools for their careers. Ultimately, this curricular development lays the groundwork for institutional enhancement of undergraduate STEM education by supporting student wellness through the engineering curriculum.
\end{abstract}

Keywords-Undergraduate education, Experiential learning, Mental health and wellness, Medical device design, Design courses, Biomedical engineering.

Address correspondence to Holly M. Golecki, Department of Bioengineering, University of Illinois Urbana-Champaign, Urbana, IL, USA. Electronic mail: golecki@illinois.edu

\section{CHALLENGE STATEMENT}

Engineering culture is dominated by perceptions of difficult and heavy workloads. This challenging environment creates a culture described as one of "suffering and shared hardship". ${ }^{14}$ Students are expected to accept demanding workloads to be successful at the expense of their mental health. ${ }^{14}$ Undergraduate mental health challenges have been increasing in prevalence in recent years. From 2007 to 2017, student mental health services utilization increased by $15 \%$ across US college campuses. ${ }^{20}$ Some studies have indicated that rates of mental health issues have been found to be higher among engineering students than non-engineering students. ${ }^{8}$ Additionally, some studies also found help-seeking to be lower for engineering students. ${ }^{20}$ These challenges create a need for colleges to provide more targeted mental health resources for students. We identified an opportunity to use data analysis and engineering design in a manner that allows faculty to promote inclusion within technical courses and students to find healthy coping methods for themselves while learning the importance of diversity in design courses.

Design courses provide an opportunity to teach engineering students the importance of achieving inclusive environments within engineering. As an engineer it is important to be able to design products that serve all populations. One need not look far to find instances of bias in engineering design. For example, the pulse-oximeter, a medical device used in the project described here, does not accurately measure blood-oxygen levels for People of Color because of the absorption-based optical measurement. ${ }^{26}$ Previous 
studies implementing Diversity, Equality, and Inclusion (DEI) principles into courses found that it helped student teams form a healthy culture as time progressed ${ }^{24}$ and understand how a person's identity impacts design and product use. ${ }^{6,15}$ Encouraging teamwork for projects in undergraduate engineering courses leads to greater idea generation by allowing each student to share their unique perspective. ${ }^{29}$

In addition to group diversity, design heuristics can help students think from more perspectives. Students in one first-year engineering course tended to revise their first design multiple times rather than try different designs. ${ }^{2}$ Design heuristics cards are a set of 77 heuristics displayed with applications of the researchbased heuristic on everyday products. ${ }^{28}$ Students can use the cards to think about features of the existing devices or their own ideas that can be improved. The goal of using the cards is to expand the design space within which the student team works by allowing them to ideate from multiple perspectives. ${ }^{23,28}$ Another study found that undergraduate students using design heuristics when analyzing and critiquing existing patent designs had more "unique" and "feasible" solutions than students who did not use design heuristics. ${ }^{1}$ Studies have found that students working in teams gain professional and technical skills ${ }^{29}$ and adapt and advance more quickly in future industry careers. ${ }^{12}$ Another study found that students' ideas of identity were based on performance of a task, while their sense of belongingness was based on peer comparison. ${ }^{25}$ First year engineering courses can utilize group projects to help students gain valuable team and social skills. Previous work suggests that engaging first year bioengineering students in a team-based, datadriven design project with an emphasis in DEI provides an opportunity to enhance student engagement when wellness is practiced in a group, to strengthen students' engineering identity through technical data analysis, and improve team skills and quality of student's designs through the use of DEI principles.

\section{NOVEL INITIATIVE}

To meet these challenges, we propose a design project that asks teams of students to practice wellness techniques over a 6-week period and use stress tracking devices to monitor biometrics while testing their own hypothesis related to how the techniques impact cardiovascular physiology. In addition to analyzing data collected before and after wellness activities, student teams look specifically at the functionality of the stress tracking devices used. Teams evaluate the functionality of the biometric measurement devices and share ideas as to how the device could be redesigned for a more accessible and enjoyable experience for users. To aid students in their development of a wellness practice, mindfulness is introduced and practiced in class, led by audio recordings or the course instructor.

Previous efforts to teach mindfulness-focused activities to engineering students included lectures, ${ }^{10,13}$ journaling, ${ }^{13}$ reflections, ${ }^{10,13}$ guided meditations ${ }^{3,10,13}$ and guest research presentations. ${ }^{13,16}$ Other studies used physical education courses, such as Gyrokinesis $^{5}$ or deep breathing exercises ${ }^{19}$ to teach wellness. A recent study by Huerta et al. developed four mindfulness workshops for students and found that students completing the workshops had improvements in mindfulness, and intrapersonal and interpersonal competencies. ${ }^{16}$ This course project aims to teach wellness topics and mindfulness techniques to students, through data collection and analysis. On the first day of class, each student receives a pulse oximeter to measure pulse rate and oxygen saturation data. Students use this data to analyze the relationship between engaging in wellness practices and the physiological effect on their own person. Fingertip pulse oximeters were chosen because they are compact, can be carried throughout the day, and are more affordable than other devices.

\section{CURRICULUM}

Bioengineering Freshman Seminar (BIOE100) is a required course for all first-year bioengineering students at University of Illinois Urbana-Champaign in which peer mentors guide students through bioengineering projects. The learning objectives of the project are (1) become familiar with the cardiovascular system, hypothesis testing, statistics, and software useful for data analysis and visualization, (2) understand and apply technologies central to the field, (3) begin independent explorations into technologies in the field, and (4) practice teamwork, technical writing, and presentations. For 5 years, the first half of the course has centered on a biometrics design project through which students explore physiology, hypothesis development, data collection and data analysis (Fig. 1). From 2016 to 2018 , after learning about cardiovascular physiology, students proposed everyday activities they hypothesized significantly impact heart rate and oxygen saturation. In a 2019 offering, $\sim 28 \%$ of students elected to study stressors (homework, or classes) or stress management techniques (meditation) within their hypothesis. The course instructor saw this as an opportunity to redesign the project and focus on studying physiological impacts of stress management techniques and use data analysis to reveal physical benefits to engineering students. In the revised offering, 


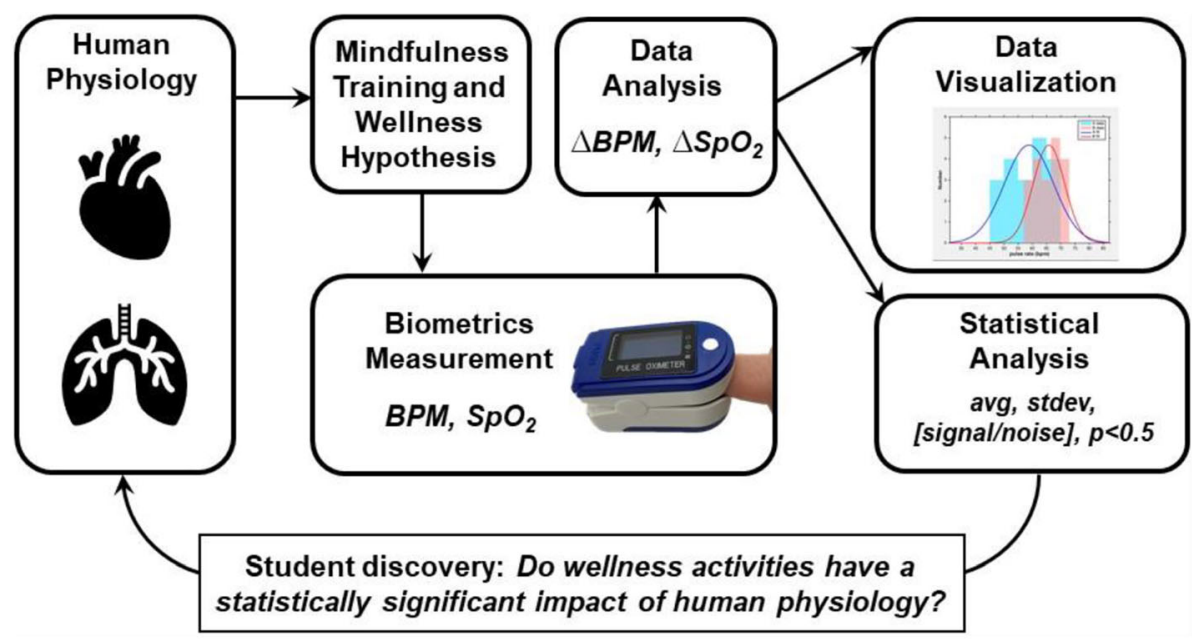

FIGURE 1. Overview of physiology and biometrics wellness project.

TABLE 1. Summary of wellness practices selected by students in course $(n=185)$.

\begin{tabular}{lc}
\hline Wellness practice & \% of teams \\
\hline Exercise & 17 \\
Music & 33 \\
Yoga & 7 \\
Meditation & 43 \\
\hline
\end{tabular}

students used mindfulness meditation or a stress management technique of their choosing (e.g., yoga, exercise, listening to calming music) to collect and analyze biometric data before and after practicing that technique. Benefits of mindfulness were introduced to students by reviewing literature on the impacts of mindfulness for students. ${ }^{9,16,21}$ Using data visualization (MATLAB) and statistical analysis (Microsoft Excel) scripts (slides used to introduce the project, data collection and analysis instructions, scripts and instructional videos provided in Supplemental Materials), students look for statistically significant changes in pulse rate and oxygen saturation as a result of the wellness techniques. Practices chosen by students are summarized in Table 1.

\section{Teaching Remotely}

Due to the COVID-19 pandemic, BIOE100 was offered fully online in Fall 2020. The class met synchronously for lecture with the instructor and in breakout rooms with peer teammates and upperclassmen mentors. Devices were mailed to students' homes and collected at the end of the semester. In Fall 2021 the course resumed in-person offerings.
In addition to focusing the project on wellness, the revised offering also included a design evaluation of existing commercially available stress tracking devices (Fig. 2). The "need" was developed through the lens of the bioengineering student: bioengineering students need a method to track changes in physiology while practicing wellness. In this case, the students themselves were the users of pulse oximeters for 6 weeks of data collection. At the end of the project, the course instructor tasked student groups with designing an improved pulse oximeter device. First, teams developed a list of features, based on their own user experience, that they would keep in a new device and features to improve. Results from this exercise are displayed in Table 2.

Within the biometrics design project, the instructor discusses engineering ethics and bias in engineering design with students. The pulse oximeter device provides an opportunity for a discussion of encoded sexism $^{7}$ and racism in design. ${ }^{4,11}$ Given the optical measurement, hemoglobin levels, and finger clip design, students use the literature probing bias in these instruments ${ }^{11}$ to discuss possible calibration methods or device redesign. The discussion focuses on design methods to correct this error, ethical responsibilities of engineers, and the benefits of a diverse engineering workforce in combatting bias in design.

Students were encouraged to use information about other commercially available stress tracking devices (examples in Fig. 2) in their analysis. Due to online learning necessitated by the COVID-19 pandemic, in Fall 2020, students learned about the devices in class but were not able to physically interact with all devices. In-person, each team of students had the opportunity to experiment with a commercially available device and report out to the class on its features and func- 


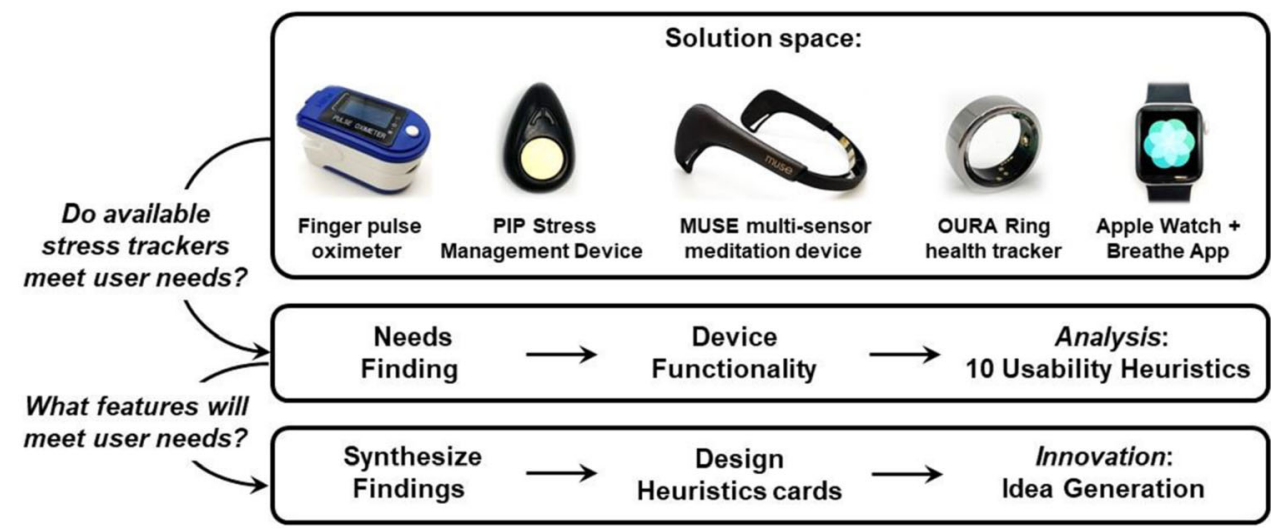

FIGURE 2. Overview of solution space and needs finding process in the pulse oximeter design project.

TABLE 2. Results from student user analysis of fingertip pulse oximeter.

\begin{tabular}{lcll}
\hline What to keep & Incidence & \multicolumn{1}{c}{ What to improve } \\
\hline Small, compact & 13 & Inconsistencies in results & Incidence \\
Easy to use & 12 & Size: bulky & 3 \\
Portable & 7 & Lag in measurement to display & 3 \\
Quick measurement & 5 & Finger needs to be still & 3 \\
Display orientation can be changed & 4 & Does not work with nail polish & 2 \\
Easy to read screen & 3 & Difficult to use if lacking fine motor skills \\
Easy on and off & 2 & Lack of calibration step & 1 \\
Long battery life (AAAs) & 1 & No on/off button & 1 \\
\hline
\end{tabular}

Students reported the features that they would keep in an improved pulse oximeter and features they would change after using the device once a day for 6 weeks.

tionality. Students analyzed devices through usability heuristics ${ }^{30}$ and propose improved device design through use of design heuristics cards. ${ }^{2,23,28}$ Table 3 shows the results of a $30 \mathrm{~min}$ brainstorming session facilitated by use the of design heuristics cards. Students were tasked with identifying three cards and specifically enumerating how the team would apply the card to redesign of the pulse oximeter.

\section{SURVEYS}

We obtained Institutional Review Board approval to administer two online surveys, a pre- and post-survey, to students enrolled in the course. Both surveys were optional to students and anonymous. To link preand post-surveys, students were asked to create a unique code. In Fall 2020, students were entered into a raffle for a $\$ 50$ gift card for each survey completed. The pre-surveys (administered at the start of the project) had a response rate of $37.9 \%$. Post-surveys (administered at the end of the semester) had a lower response rate of $17.2 \%$. The pre-survey asked questions about student's perception of, practice of, and willingness to try mindfulness. ${ }^{22}$ Students were asked again about their perception and practice of mindfulness on the post-survey to gauge changes after the project. The post-survey included additional questions about the quality of the project and devices, and usefulness of the devices in helping students to identify stress, and effectiveness of the devices in gaining a better understanding of design heuristics. In Fall 2021, the survey was offered in person during class time and compensation was changed to incentivize participation. Participants were paid $\$ 10$ per survey completed. These changes resulted in a pre-survey response rate of $97 \%$ and a post-survey response rate of $60 \%$.

\section{PILOT ASSESSMENT}

A pilot of the new project was first implemented in Fall 2020 with approximately 90 first-year engineering students. The pilot offering (1) introduced students to new biometric devices that capture additional physiological data including electrodermal activity and EEG (Fig. 2) for design comparison, (2) introduced students to the concept of wellness, and (3) collected general student feedback on the project implementation to improve future offerings. The pilot project was suc- 
TABLE 3. Example student responses to application of design heuristics cards to redesign of the pulse oximeter.

Card number and title

How the card would be applied

4. Add to existing product "The pulse oximeter could have a [tare] function similar to a balance. After taking your measurements once, you could set the pulse oximeter to show you the difference the second time after doing any activity."

"I think that one thing that makes a device like the Apple Watch so popular is that it integrates the high-tech pulse oximeter into an already existing, extremely smart piece of technology that has many other functions as well. The watch idea, for example, fixes the mechanical issue of having a large, plastic device and instead makes using the pulse oximeter a trendy aspect of a new tech giant's product."

"Since the pulse oximeter is only one datapoint in the scope of a person's health, creating a system of vital sign detectors could give a broader look at the status of one's health. For example, by connecting a pulse oximeter to a thermometer, a blood pressure monitor, or a nebulizer, one can use the pulse oximeter in a more meaningful way."

9. Allow user to customize

"Allow the user to customize settings about themselves, which can lead to a more accurate reading. For instance, users can enter their skin pigmentation and the pulse oximeter can make adjustments to the measurements to more accurately represent the actual $\mathrm{SpO}_{2}$ value."

"I think it would be nice if you could store recent data in the pulse oximeter. Like your 10 most recent data measurements so you could see if there are any concerning patterns."

11. Allow user to reorient "If the pulse oximeter was perpendicular to your finger, people with nail polish on or acrylic nails could still use it."

15. Attach product to user "Attach pulse oximeter to a watch or some wearable device so the user can monitor their pulse rate and oxygen saturation throughout the mindfulness practice."

17. Build user community "The pulse oximeter could have bigger computational components that would allow for software to be implemented that would allow users to connect with each other via an app on a smartphone. In the app, you could compare your heart rate and SpO2 levels to your friends, thus monitoring their workouts, meditation, etc."

19. Change flexibility

"If the pulse oximeter was made out of a soft, gel-like or clay-like material such as the grip on an ergonomic pencil, the oximeter would become more patient friendly because the device would mold to the patient's finger and it would be less bulky."

27. Cover or wrap

"Implement a wrapping mechanism to allow for greater flexibility and account for different finger shapes and sizes."

"For groups that used the pulse oximeter while doing exercise, a pulse oximeter that wraps around an individual's arm may be more secure than the current design."

40. Incorporate user input "Input user resting heart rate so the device can compare your data after practicing mindfulness."

43. Make multifunctional "We could make the device measure another biometric, such as cortisol, through sweat. By measuring cortisol, we could also measure the individual's stress level."

50. Provide sensory feedback

"When reading is in the process of being taken, animation is shown so the user knows the device is thinking. When reading is complete, the device vibrates to let the user know it has finished."

"You could allow the pulse oximeter to keep track of average values and alert you when the measurements that you are getting are abnormal or cause for concern. This way, people could keep track of their health better and take action when something is wrong."

70. Use different energy "A USB-C port or a wireless charging driver would be a very useful addition to the device." source

"Instead of using batteries as energy, could make it solar powered or powered by charging it with an outlet."

cessful as measured by qualitative measures and course feedback surveys. Students noted that collecting their own biometric data was convincing of the effectiveness of wellness practices (Table 4). Students noted that the project applied engineering principles while also providing students valuable life skills. Students also indicated that the project was useful in learning about engineering design.

Students also provided suggestions for improvements to the project. Some of the pulse oximeters used by students were unreliable, so students suggested getting better or newer devices for the class. One student suggested that more wellness practices "that aren't just mindfulness activities or light exercise" could be introduced. We also gathered feedback on the usefulness of pulse oximeters during the wellness pro- ject. Eight students reported that using the pulse oximeters helped them become more aware of stress, five students said they did not, and one student did not use the device in this way. After Fall 2020 and Fall 2021 offerings, 68 students reported that pulse oximeters were useful in improving their understanding of design features (Table 5). One student reported they did not gain a better understanding of design from using the pulse oximeters but did not further elaborate in a comment.

\section{REFLECTION}

Overall receptiveness to the wellness project, as identified from the post survey, was positive. Students noted enjoying designing their own experiment related 
TABLE 4. Themes from student responses about what they enjoyed about the wellness project (Year 1).

\begin{tabular}{|c|c|c|}
\hline Theme & $\begin{array}{l}\text { Number of } \\
\text { responses }\end{array}$ & Example student comments \\
\hline Analyzing own data & 2 & $\begin{array}{l}\text { "I enjoyed how we could analyze data from our own experiences, which also led to some } \\
\text { benefits of our own." } \\
\text { "I liked that I was able to see a measurable change in my data, because it reinforced to me that } \\
\text { mindfulness does in fact work." }\end{array}$ \\
\hline Way to de-stress & 5 & $\begin{array}{l}\text { "It made me take time out of my day to slow down." } \\
\text { "I liked that it forced me to take some time out of my day to just relax." }\end{array}$ \\
\hline $\begin{array}{l}\text { Applicable outside of } \\
\text { academics }\end{array}$ & 2 & $\begin{array}{l}\text { "I liked how useful and applicable it was to my life." } \\
\text { "I liked how the project was relevant to both bioengineering and to us as students." }\end{array}$ \\
\hline $\begin{array}{l}\text { Freedom to create own } \\
\text { project }\end{array}$ & 3 & $\begin{array}{l}\text { "I liked the freedom of choosing what to incorporate as a wellness practice." } \\
\text { "The freedom aspect of being able to choose what mindfulness activity to engage in." }\end{array}$ \\
\hline
\end{tabular}

TABLE 5. Student feedback on usefulness of pulse oximeters in gaining a better understanding of design features (Years 1 and 2 ).

\begin{tabular}{|c|c|}
\hline Response & Number of responses \\
\hline Yes & 68 \\
\hline No & 5 \\
\hline $\begin{array}{l}\text { Example student com- } \\
\text { ments }\end{array}$ & $\begin{array}{l}\text { "Yes, being able to have a medical device and use it personally gave me a lot of insight into the design and use } \\
\text { processes." } \\
\text { "Yes, it gave me a better sense of the features such as pulse rate and oxygen levels that can help track stress levels } \\
\text { for people." } \\
\text { "Using the biometric devices and talking with my group about the issues we encountered when using them gave us } \\
\text { a better sense of how to improve these devices." } \\
\text { "I realized we need to better diversify the biometric devices we use since they did have bias within their design." }\end{array}$ \\
\hline
\end{tabular}

to wellness and using biometric devices. A difficulty that arose during the project concerned the quality of the pulse oximeter devices. In future offerings we plan to improve the project by supplying students with higher quality pulse oximeters that give more consistent readings, as well as allowing students to check-out other devices shown in Fig. 2. In teams, students can further compare the design and functionality of different devices, identify needs, and posit solutions for improvement. In Fall 2021, the instructor led live inperson mindfulness meditations. At the end of the 6week project, $88 \%$ of students in the course voted to continue meditating daily at the start of class. We plan to collect data and assess changes in help-seeking, perceived barriers to care, and use of university wellness resources in future offerings, ultimately to shift the engineering culture from one of "suffering and shared hardship" 14 to a culture of wellness. ${ }^{17}$

\section{SUPPLEMENTARY INFORMATION}

The online version contains supplementary material available at https://doi.org/10.1007/s43683-021-000601.

\section{ACKNOWLEDGMENTS}

The authors thank Professor Andrew Smith for the resources originally developed for the BIOE100 course and the students for their feedback.

\section{FUNDING}

This work was supported by the Department of Bioengineering at the University of Illinois UrbanaChampaign and the University of Illinois UrbanaChampaign Faculty Retreat Grant.

\section{DATA AVAILABILITY}

All materials are available on our website, https:// www.hollygolecki.com/teaching or upon request.

\section{CODE AVAILABILITY}

MATLAB code for this project is available for download on, https://www.hollygolecki.com/teaching or upon request. Scripts and instructional videos for this project are available for download on https://ww w.hollygolecki.com/teaching and upon request. 


\section{CONSENT FOR PUBLICATION}

Not applicable.

\section{CONFLICT OF INTEREST}

The authors declare no conflict of interest.

\section{ETHICAL APPROVAL}

This work is approved by UIUC IRB \#21171 and 21782.

\section{INFORMED CONSENT}

Not applicable.

\section{REFERENCES}

${ }^{1}$ Altshuller GS. Creativity as an exact science. CRC Press; 1984. https://doi.org/10.1201/9781466593442.

${ }^{2}$ Ayer SK, Messner JI, Anumba CJ. Challenges and benefits of open-ended sustainable design in first-year engineering. J Prof Issues Eng Educ Pract. 2014;140(2):04013017. http s://doi.org/10.1061/(ASCE)EI.1943-5541.0000192.

${ }^{3}$ Bellinger DB, DeCaro MS, Ralston PAS. Mindfulness, anxiety, and high-stakes mathematics performance in the laboratory and classroom. Conscious Cogn. 2015;37:12332. https://doi.org/10.1016/j.concog.2015.09.001.

${ }^{4}$ Bickler PE, Feiner JR, Severinghaus JW. Effects of skin pigmentation on pulse oximeter accuracy at low saturation. Anesthesiology. 2005;102(4):715-19. https://doi.org/10.10 97/00000542-200504000-00004.

${ }^{5}$ Caldwell K, Harrison M, Adams M, Quin RH, Greeson J. Developing mindfulness in college students through movement-based courses: effects on self-regulatory selfefficacy, mood, stress, and sleep quality. J Am Coll Health. 2010;58(5):433-42. https://doi.org/10.1080/0744848090354 0481 .

${ }^{6}$ Carpenter A, et al. Pilot study: impact of social consciousness on engineering design decision making. In: Presented at the American Society of Engineering Education, July 2021

${ }^{7}$ Criado-Perez C. Invisible women: data bias in a world designed for men. New York: Abrams Press; 2019.

${ }^{8}$ Danowitz A, Beddoes K. Characterizing mental health and wellness in students across engineering disciplines. In: 2018 CoNECD - Collaborative Network for Engineering and Computing Diversity conference, April 2018. https://jee.o rg/29522. Accessed 14 Dec 2021.

${ }^{9}$ Delizonna LL, Williams RP, Langer EJ. The effect of mindfulness on heart rate control. J Adult Dev. 2009;16(2):61-5. https://doi.org/10.1007/s10804-009-90506.

${ }^{10}$ Estrada T, Dalton E. Impact of student mindfulness facets on engineering education outcomes: an initial exploration. In: 2019 ASEE annual conference and exposition proceedings, Tampa, Florida, June 2019, p. 32934. https://doi. org/10.18260/1-2-32934.
${ }^{11}$ Feiner JR, Severinghaus JW, Bickler PE. Dark skin decreases the accuracy of pulse oximeters at low oxygen saturation: the effects of oximeter probe type and gender. Anesth Analg. 2007;105(Online Supplement):S18-23. http s://doi.org/10.1213/01.ane.0000285988.35174.d9.

${ }^{12}$ Fowler W. Teaming in engineering design courses. Charlotte, June 1999, pp. 4.498.1-6. https://peer.asee.org/7983. Accessed 14 Dec 2021.

${ }^{13} \mathrm{Ge}$ J, Berger E, Major J, Froiland J. Teaching undergraduate engineering students gratitude, meaning, and mindfulness. In: 2019 ASEE annual conference and exposition proceedings, Tampa, Florida, June 2019, p. 33358. https://d oi.org/10.18260/1-2-33358.

${ }^{14}$ Godfrey E, Parker L. Mapping the cultural landscape in engineering education. J Eng Educ. 2010;99(1):5-22. http s://doi.org/10.1002/j.2168-9830.2010.tb01038.x.

${ }^{15}$ Hedayati Mehdiabadi A, Atadero R, Baker D, Casper AM. The effects of infusing diversity and inclusion into a design problem in engineering mechanics: statics. In: 2019 ASEE annual conference and exposition proceedings, Tampa, Florida, June 2019, p. 33387. https://doi.org/10.18260/1-233387.

${ }^{16}$ Huerta M. Inner engineering: a convergent mixed methods study evaluating the use of contemplative practices to promote resilience among freshman engineering students. In: 2018 ASEE annual conference and exposition proceedings, Salt Lake City, Utah, June 2018, p. 30664. http s://doi.org/10.18260/1-2-30664.

${ }^{17} \mathrm{Jensen} \mathrm{K}$. The time is now to build a culture of wellness in engineering. Stud Eng Educ. 2021;2(2):42. https://doi.org/ 10.21061 /see.67.

${ }^{18}$ Jensen KJ, Cross KJ. Engineering stress culture: relationships among mental health, engineering identity, and sense of inclusion. J Eng Educ. 2021;110(2):371-92. https://doi. org/10.1002/jee.20391.

${ }^{19} \mathrm{Kim}$ B-Y, Bae M-J, Bae S-G. A study on reducing stress through deep breathing. Int J Appl Eng Res. 2018;13(2):1460-4.

${ }^{20}$ Lipson SK, Lattie EG, Eisenberg D. Increased rates of mental health service utilization by U.S. college students: 10-year population-level trends (2007-2017). Psychiatr Serv. 2019;70(1):60-3. https://doi.org/10.1176/appi.ps.201 800332.

${ }^{21}$ Miller I, Jensen K. Introduction of mindfulness in an online engineering core course during the COVID-19 pandemic. Adv Eng Educ. 2020;8(4, Fall). https://advances.asee.org/ wp-content/uploads/Covid\%2019\%20Issue/Text/AEE-CO VID-19-Jensen.pdf. Accessed 14 Dec 2021.

${ }^{22}$ Miller I, Lamer S, Jensen K, Golecki H. Work in progress: supporting students mental health: understanding the use of biometrics analysis in an engineering design project to promote wellness. In: ASEE virtual annual conference, July 2021.

${ }^{23}$ Ostrowski A, Lee JW, Daly S, Huang-Saad A, Seifert C. Design in biomedical engineering: student applications of design heuristics as a tool for idea generation. In: 2017 ASEE annual conference and exposition proceedings, Columbus, Ohio, June 2017, p. 28115. https://doi.org/10. 18260/1-2-28115.

${ }^{24}$ Rambo-Hernandez K, Morris M, Casper AM, Hensel R, Schwartz J, Atadero R. Examining the effects of equity, inclusion, and diversity activities in first-year engineering classes. In: 2019 ASEE annual conference and exposition proceedings, Tampa, Florida, June 2019, p. 32782. https://d oi.org/10.18260/1-2-32782. 
${ }^{25}$ Rohde J, et al. Design experiences, engineering identity, and belongingness in early career electrical and computer engineering students. IEEE Trans Educ. 2019;62(3):165-72. https://doi.org/10.1109/TE.2019.2913356.

${ }^{26}$ Sjoding MW, Dickson RP, Iwashyna TJ, Gay SE, Valley TS. Racial bias in pulse oximetry measurement. N Engl J Med. 2020;383(25):2477-8. https://doi.org/10.1056/NEJMc 2029240.

${ }^{27}$ Toh CA, Miller SR. Creativity in design teams: the influence of personality traits and risk attitudes on creative concept selection. Res Eng Des. 2016;27(1):73-89. https://d oi.org/10.1007/s00163-015-0207-y.

${ }^{28}$ Yilmaz S, Daly SR, Seifert CM, Gonzalez R. Evidencebased design heuristics for idea generation. Des Stud.
2016;46:95-124. https://doi.org/10.1016/j.destud.2016.05.0 01 .

${ }^{29}$ Zarske M, Yowell J, Maierhofer S, Reamon D. Teamwork in first-year engineering projects courses: does training students in team dynamics improve course outcomes and student experiences? In: 2013 ASEE annual conference and exposition proceedings, Atlanta, Georgia, June 2013, pp. 23.1159.1-15. https://doi.org/10.18260/1-2-22544.

${ }^{30}$ Zhang J, Johnson TR, Patel VL, Paige DL, Kubose T. Using usability heuristics to evaluate patient safety of medical devices. J Biomed Inform. 2003;36(1-2):23-30. h ttps://doi.org/10.1016/S1532-0464(03)00060-1. 